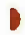

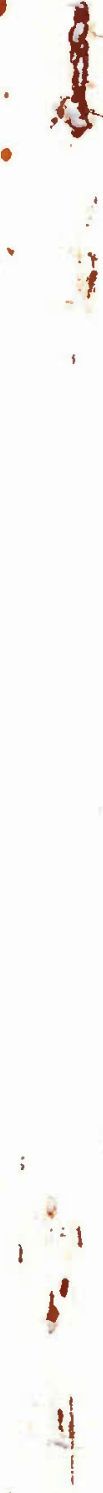

\title{
PHEROMONE CHIRALITY OF AFRICAN PALM WEEVIL, Rhynchophorus phoenicis (F.) AND PALMETTO WEEVIL, Rhynchophorus cruentatus (F.) (COLEOPTERA: CURCULIONIDAE)
}

\author{
ALICE L. PEREZ,' GERHARD GRIES, ${ }^{2}$ REGINE GRIES ${ }^{2}$ \\ ROBIN M. GIBLIN-DAVIS, ${ }^{3}$ and A. CAMERON OEHLSCHLAGER ${ }^{1, *}$ \\ 'Department of Chemistry \\ ${ }^{2}$ Centre for Pest Management, Department of Biological Sciences \\ Simon Fraser University \\ Burnaby, British Columbia, Canada V5A 1 S6 \\ ${ }^{3}$ Institute of Food and Agricultural Sciences \\ Fort Lauderdale Research and Education Centre \\ University of Florida, Fort Lauderdale, Florida 3314
}

(Received January 10, 1994; accepted June 2, 1994)

\begin{abstract}
There are four stereoisomers of both 3-methyl-octan-4-ol, the aggregation pheromone of the African palm weevil, Rhynchophorus phoenicis (F.) and 5-methyl-octan-4-ol, the aggregation pheromone of the palmetto weevil, Rhynchophorus cruentatus (F.). Synthetic stereoisomers of 3-methyl-octan4-ol and 5-methyl-octan-4-ol were baseline-separated on a Cyclodex-B fused silica column. Use of this column in gas chromatographic-electroantennographic detection (GC-EAD) and GC-mass spectrometric (GC-MS) analyses revealed that only one stereoisomer, $(3 S, 4 S)$-3-methyl-octan-4-ol and $(4 S, 5 S)$ 5-methyl-octan-4-ol, is produced by male $R$. phoenicis and male $R$. cruentatus, respectively, and elicits good antennal responses by conspecific male and female weevils. In field trapping experiments, with $R$. phoenicis in Côte d'Ivoire and $R$. cruentatus in Florida, $(3 S, 4 S)$-3-methyl-octan-4-ol and $(4 S, 5 S)$-5-methyl-octan-4-ol strongly enhanced attraction of fresh palm tissue, whereas other stereoisomers were behaviorally benign. Stereoisomeric 3-methyl-octan-4-ol and 5-methyl-octan-4-ol may be utilized to monitor and/ or manage populations of these two palm weevils.
\end{abstract}

Key Words-Coleoptera, Curculionidae, Rhynchophorus phoenicis, Rhynchophorus cruentatus, aggregation pheromone, pheromone chirality, $(3 S, 4 S)$ 3-methyl-octan-4-ol, $(3 R, 4 R)$-3-methyl-octan-4-ol, $(3 S, 4 R)$-3-methyl-octan-4-

*To whom correspondence should be addressed. 
ol, $\quad(3 R, 4 S)$-3-methyl-octan-4-ol, $\quad(4 S, 5 S)$-5-methyl-octan-4-ol, $\quad(4 R, 5 R)-5$ methyl-octan-4-ol, $(4 S, 5 R)$-5-methyl-octan-4-ol, $(4 R, 5 S)$-5-methyl-octan-4-ol.

\section{INTRODUCTION}

Palm weevils in the Rhynchophorinae produce methyl-branched, secondary alcohols as aggregation pheromones: (2E)-6-methyl-hepten-4-ol (rhynchophorol) [American palm weevil, Rhynchophorus palmarum (L.) (Rochat et al., 1991)]; 3-methyl-octan-4-ol (phoenicol) [African palm weevil, $R$. phoenicis (F.) (Gries et al., 1993, 1994; Rochat et al., 1993]; 4-methyl-nonan-5-ol (ferrugineol) [Asian palm weevils, $R$. ferrugineus (Oliv.), $R$. vulneratus (Panz) Hallett et al., 1993; Rochat et al., 1993) and $R$. bilineatus (Montr.) (Oehlschlager et al., 1994)], and 5-methyl-octan-4-ol (cruentol) [Palmetto weevil, $R$. cruentatus (F.) (Weissling et al., 1994)]. Racemic (rhynchophorol) and stereoisomeric mixtures (phoenicol, ferrugineol, cruentol) of synthetic aggregation pheromones in combination with host material strongly attracted weevils in field experiments. Stereoselective production of and response to pheromone has been demonstrated in R. palmarum (Oehlschlager et al., 1992) and recently in the other Rhynchorphorus palm weevils (Perez et al., 1993). Male R. palmarum stereoselectively produce and both sexes respond to $(S)$-rhynchophorol, while the antipode is behaviorally benign (Oehlschlager et al., 1992). Male $R$. phoenicis produce one stereoisomer of phoenicol but electrophysiological and behavioral activity have not been investigated (Mori et al., 1993). In this study we report that $R$. phoenicis and $R$. cruentatus stereoselectively produce and respond to only one of the four possible stereoisomers of 3-methyl-octan-4-ol and 5-methyl-octan4-ol, respectively.

\section{METHODS AND MATERIALS}

\section{Laboratory Analysis}

Male and female $R$. phoenicis were collected in oil palm plantations 40-50 km northeast of Abidjan, Côte d'Ivoire. Male and female $R$. cruentatus were collected in a 300-ha pasture interspersed with Sabal palmetto (Walter) and saw palmetto, Serrenoa repens (Bartr.), $12 \mathrm{~km}$ south of La Belle, Florida. Male-produced phoenicol and cruentol were captured (Gries et al., 1993; Weissling et al., 1994) and subjected to both gas chromatographic-electroantennographic detection (GC-EAD) (Arn et al., 1975) (Hewlett Packard 5890A) and GC-mass spectrometry (GC-MS) (Hewlett Packard 5985 B) on a fused silica, Cyclodex-B-coated column ( $30 \mathrm{~m} \times 0.25 \mathrm{~mm} \mathrm{ID}$, J\&W Scientific), which separates all four stereoisomers of phoenicol and cruentol. For GC-EAD recordings, a weevil antenna was removed from the rostrum and suspended between 
two glass capillary electrodes with the antennal base being inserted into one and the olfactory club impaled by the other electrode. Chemical ionization (CI, isobutane) GC-MS analysis was conducted in both full-scan and selected-ion monitoring mode (SIM). A full-scan mass spectrum of synthetic phoenicol or cruentol was obtained to select diagnostic ions. For GC-MSCI-SIM, synthetic phoenicol and cruentol, hexane, and concentrated weevil-produced pheromone were injected in split mode and analyzed by scanning for diagnostic ions.

\section{Instruments and General Procedures}

Nuclear magnetic resonance (NMR) spectroscopy was conducted on a Bruker AMX-400 spectrometer at 400.13 and $100.62 \mathrm{MHz}$ for ${ }^{1} \mathrm{H}$ and ${ }^{13} \mathrm{CNMR}$ spectra, respectively. ${ }^{1} \mathrm{H}$ chemical shifts are reported in parts per million (ppm, $\delta)$ and relative to TMS $(0.00 \mathrm{ppm}) .{ }^{13} \mathrm{C}$ spectra are referred to $\mathrm{CDCI}_{3}(77.0$ ppm). Gas chromatographic analyses were performed on Hewlett-Packard 5880A and 5890 instruments equipped with a flame ionization detector and a fused silica, DB-1 coated column ( $15 \mathrm{~m} \times 0.25 \mathrm{~mm}$ ID; $0.25 \mu \mathrm{m}$ film) (J\&W Scientific). Elemental analyses were performed using a Carbo Erba model-1106 Elemental Analyzer. Diethyl ether $\left(\mathrm{Et}_{2} \mathrm{O}\right)$, dichloromethane $\left(\mathrm{CH}_{2} \mathrm{Cl}_{2}\right)$, and pentane were freshly distilled from sodium-benzophenone-ketyl, $\mathrm{CaH}$, and $\mathrm{P}_{2} \mathrm{O}_{5}$, respectively. Chemicals obtained from commercial sources were used without further purification unless otherwise indicated. All moisture and air sensitive reactions were conducted under argon. Column chromatography refers to flash chromatography using Silica Gel 60 (230-400 mesh E Merck, Darmstadt) (Still et al., 1978). Thin-layer chromatography (TLC) was conducted on aluminumbacked plates precoated with Merck Silica Gel 60F-254 as the adsorbent, and visualized by treatment with an acidic solution of $1 \% \mathrm{Ce}\left(\mathrm{SO}_{4}\right)_{2}$ and $1.5 \%$ molybdic acid followed by gentle heating.

\section{Synthesis of Phoenicol Stereoisomers}

$(3 R, 4 R)-,(3 S, 4 S)-,(3 R, 4 S)$-, and $(3 S, 4 R)-3$-methyl-octan-4-ol [ $(R, R)$-, $(S, S)$-, $(R, S)$-, and $(S, R)$-phoenicol] were synthesized according to a method modified from Nakagawa and Mori (1984), which involved: (1) asymmetric epoxidation of (2Z-) or (2E)-2-penten-1-ol (Gao et al., 1987; Hill et al., 1985); (2) regioselective epoxide opening with trimethylaluminun (Pfaltz and Mattenberger, 1982; Suzuki et al., 1982; Takano et al., 1989; Vaccaro et al., 1992); (3) selective monotosylation; and (4) alkylation reaction using an organomagnesium cuprate reagent. Synthesis of $(3 S, 4 S)$-3-methyl-octan-4-ol exemplifies the synthetic procedure (Figure 1):

(2S,3R)-2,3-Epoxy-pentan-1-ol (2a). Titanium(IV) isopropoxide (11.4 ml, $10.87 \mathrm{~g}, 38 \mathrm{mmol}$ ) in $250 \mathrm{ml}$ of dry $\mathrm{CH}_{2} \mathrm{Cl}_{2}$ was mixed under argon with $1 \mathrm{~g}$ of $4 \mathrm{~A}$ powdered, activated molecular sieves. After cooling to $-78^{\circ} \mathrm{C}$, diethyl 


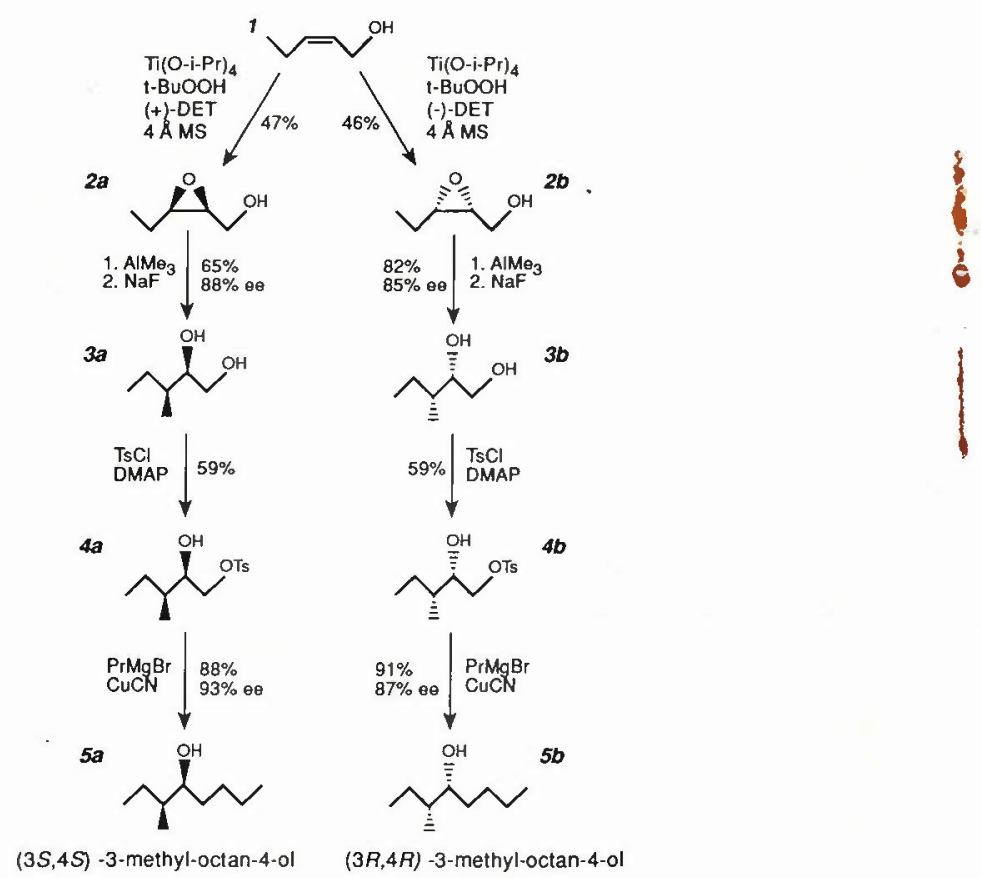

FrG. 1. Scheme for the synthesis of $(3 S, 4 S)$-3-methyl-octan-4-ol, 5a, and $(3 R, 4 R)-3$ methyl-octan-4-ol, 5 b.

$(2 R, 3 R)$-tartrate [L-(+)-DET, $7.8 \mathrm{ml}, 9.9 \mathrm{~g}, 0.05 \mathrm{~mol}]$ was added via syringe followed by addition of (2Z)-2-penten-1-ol $(8.21 \mathrm{ml}, 7.0 \mathrm{~g}, 80 \mathrm{mmol}$, Aldrich Chemical Co., Milwaukee, Wisconsin). The mixture was stirred 15 min prior to dropwise addition of $25 \mathrm{ml}(0.15 \mathrm{~mol})$ of $5.7 \mathrm{M}$ anhyd. tert-butyl hydroperoxide in $\mathrm{CH}_{2} \mathrm{Cl}_{2}$ (prepared as described by Gao et al., 1987) (precooled to $-20^{\circ} \mathrm{C}$ ). After the reaction had warmed up to $-20^{\circ} \mathrm{C}$ it was stirred at this temperature for $48 \mathrm{hr}$. The reaction was monitored by TLC $\left(2: 8\right.$, pentane- $\mathrm{Et}_{2} \mathrm{O}$; $R_{f}=0.39$ ). Nonaqueous work-up (Gao et al., 1987) followed by column chromatography $\left(2: 8\right.$, pentane- $\left.\mathrm{Et}_{2} \mathrm{O}\right)$ gave $2 \mathrm{a}(3.79 \mathrm{~g}, 46 \%$ yield, $90 \%$ ee) as a colorless liquid [2a: ${ }^{1} \mathrm{H}\left(\mathrm{CDCl}_{3}\right): \delta 1.02(3 \mathrm{H}, \mathrm{t}, J=8.6 \mathrm{~Hz}), 1.52(2 \mathrm{H}, \mathrm{m})$, $2.04\left(1 \mathrm{H}\right.$, brs, $\mathrm{D}_{2} \mathrm{O}$ exchangeable $), 2.41(2 \mathrm{H}, \mathrm{t}, J=10 \mathrm{~Hz}), 3.15(1 \mathrm{H}$, dd, $J=5,10 \mathrm{~Hz}), 3.68(1 \mathrm{H}, \mathrm{dd}, J=4,10 \mathrm{~Hz}) ;{ }^{13} \mathrm{C}\left(\mathrm{CDCl}_{3}\right) \delta 61.82,57.10$, $56.98,26.22,13.91 \mathrm{ppm})] .2 \mathrm{~b}(3.93 \mathrm{~g}, 47 \%$ yield, $87 \%$ ee) was synthesized following the same procedure using diethyl $(2 S, 3 S)$-tartrate [D-(-)-DET] as the epoxidation catalyst.

(2R,3S)-3-Methyl-pentane-1,2-diol (3a). A pentane (200 $\mathrm{ml}$ solution of 2a $(3.78 \mathrm{~g}, 37 \mathrm{mmol})$ was cooled to $-50^{\circ} \mathrm{C}$. Then $10.5 \mathrm{ml}(0.11 \mathrm{~mol})$ of 
neat $\mathrm{AlMe}_{3}$ (Aldrich Chemical Co.) was added dropwise followed by $8 \mathrm{ml}$ of $2.49 \mathrm{M} n$-butyllithium $(20 \mathrm{mmol})$. After stirring at $-50^{\circ} \mathrm{C}$ for $20 \mathrm{~min}$., the cooling bath was removed and the flask allowed to warm to room temperature. Monitoring the reaction by GC and TLC $\left(1: 9\right.$, pentane- $\left.\mathrm{Et}_{2} \mathrm{O} ; R_{f}=0.19\right)$ indicated reaction completion after $30 \mathrm{~min}$. The reaction was quenched with $\mathrm{NaF}-$ $\mathrm{H}_{2} \mathrm{O}(1: 1)$ (Suzuki et al., 1982) at $0^{\circ} \mathrm{C}$. The white precipitate was filtered and the obtained solid was washed with $\mathrm{Et}_{2} \mathrm{O}$. The ethereal layer was dried over anhyd. $\mathrm{MgSO}_{4}$ and concentrated in vacuo to give a pale yellow liquid. Purification by column chromatography $\left(1: 9\right.$, pentane- $\left.\mathrm{Et}_{2} \mathrm{O}\right)$ afforded $3 \mathrm{a}(2.93 \mathrm{~g}$, $64.4 \%$ yield, $88 \%$ ee) as a colorless liquid [3a: ${ }^{1} \mathrm{H}\left(\mathrm{CDCl}_{3}\right) ; \delta 0.88(3 \mathrm{H}, \mathrm{t}, J$ $=8 \mathrm{~Hz}), 0.90(3 \mathrm{H}, \mathrm{d}, J=8 \mathrm{~Hz}), 1.20(1 \mathrm{H}, \mathrm{m}), 1.42(2 \mathrm{H}, \mathrm{m}), 2.15(2 \mathrm{H}$, brs, $\mathrm{D}_{2} \mathrm{O}$ exchangeable $), 3.45(2 \mathrm{H}, \mathrm{m}), 3.62(1 \mathrm{H}, \mathrm{m}) ;{ }^{13} \mathrm{C}\left(\mathrm{CDCl}_{3}\right) \delta 75.52$, $65.21,37.40,25.71,14.11,11.53 \mathrm{ppm}$; CI-MS $\mathrm{m} / \mathrm{z}$ (isobutane, relative intensity): $119\left(\mathrm{M}^{+}+1,40\right)$.] 3b: $3.59 \mathrm{~g}, 82 \%$ yield, $85 \%$ ee.

(2R,3S)-3-Methyl-1-tosyloxy-pentan-2-ol (4a). This was prepared from $2.92 \mathrm{~g}(25 \mathrm{mmol})$ of $3 \mathrm{a}$ in dry pyridine, to which $0.73 \mathrm{~g}(6 \mathrm{mmol})$ of dimethyl aminopyridine (DMAP) was added. The flask was cooled to $-20^{\circ} \mathrm{C}$ (ethylene glycol-water-Dry Ice) and $5.73 \mathrm{~g}(0.03 \mathrm{~mol})$ of $p$-toluensulfonyl chloride added in one portion. After stirring $7 \mathrm{hr}$ at -20 to $-10^{\circ} \mathrm{C}$ and monitoring the reaction by $\mathrm{GC}$ and TLC $\left(6: 4\right.$, pentane- $\left.\mathrm{Et}_{2} \mathrm{O}, R_{f}=0.27\right)$, the mixture was poured into ice-cooled $\mathrm{NaCl}$ solution and extracted $(2 \times 30 \mathrm{ml})$ with $\mathrm{Et}_{2} \mathrm{O}$. The organic layer was washed with $3 \mathrm{M} \mathrm{HCl}$, saturated $\mathrm{NaHCO}_{3}$, saturated $\mathrm{NaCl}$, and dried over anhyd. $\mathrm{MgSO}_{4}$. After concentration and column chromatography $(6: 4$, pentane- $\left.\mathrm{Et}_{2} \mathrm{O}\right)$, solvent residues were removed under vacuum to give $4 \mathrm{a}(4.0 \mathrm{~g}$, $59 \%)$ as a pale yellow oil. [4a: ${ }^{1} \mathrm{H}\left(\mathrm{CDCl}_{3}\right) ; \delta 0.85(3 \mathrm{H}, \mathrm{d}, J=8 \mathrm{~Hz}), 0.86$ $(3 \mathrm{H}, \mathrm{t}, J=8 \mathrm{~Hz}), 1.20(1 \mathrm{H}, \mathrm{m}), 1.45(2 \mathrm{H}, \mathrm{m}), 1.90\left(1 \mathrm{H}, \mathrm{brs}, \mathrm{D}_{2} \mathrm{O}\right.$ exchangeable), $3.72(1 \mathrm{H}, \mathrm{dt}, J=8,4 \mathrm{~Hz}), 3.98(1 \mathrm{H}, \mathrm{dd}, J=8,2.5 \mathrm{~Hz})$, $4.04(1 \mathrm{H}, \mathrm{dd}, J=8,1.5 \mathrm{~Hz}) ; 7.34(2 \mathrm{H}, \mathrm{d}, J=8 \mathrm{~Hz}) ; 7.80(2 \mathrm{H}, \mathrm{d}, J=$ $8 \mathrm{~Hz}) ;{ }^{13} \mathrm{C}\left(\mathrm{CDCl}_{3}\right) ; \delta 144.95,133.0,129.89,127.90,72.93,72.75,36.97$, $25.62,21.57,13.57,11.41 \mathrm{ppm}$; CI-MS $m / z$ (relative intensity): $273\left(\mathrm{M}^{+}+1\right.$, 100).] 4b: $4 \mathrm{~g}, 59 \%$ yield.

(3S,4S)-3-Methyl-octan-4-ol (5a). This was prepared from propyl magnesium bromide $(0.17 \mathrm{~mol})$ in dry $\mathrm{Et}_{2} \mathrm{O}$ [prepared by Grignard reaction between $n$-propyl bromide (Aldrich Chemical Co.) and magnesium turnings], which was cooled to $-40^{\circ} \mathrm{C}$ and $1.58 \mathrm{~g}(17 \mathrm{mmol})$ of $\mathrm{CuCN}$ were added. After stirring the mixture for $30 \mathrm{~min}$, the flask was cooled to $-78^{\circ} \mathrm{C}$ and $4.89 \mathrm{~g}(17 \mathrm{mmol})$ of $4 \mathrm{a}$ in $25 \mathrm{ml}$ of dry $\mathrm{Et}_{2} \mathrm{O}$ added via cannula. After $30 \mathrm{~min}$ of stirring, the cold bath was removed and the reaction allowed to warm to room temperature. The course of the reaction was followed by GC and TLC $\left(9: 1\right.$, pentane- $\mathrm{Et}_{2} \mathrm{O}, R_{f}$ $=0.58$ ). Upon completion, the reaction was quenched with $3 \mathrm{M} \mathrm{HCl}$ at $0^{\circ} \mathrm{C}$. The aqueous layer was extracted with $\mathrm{Et}_{2} \mathrm{O}(3 \times 25 \mathrm{ml})$, washed with both saturated $\mathrm{NaHCO}_{3}$ and $\mathrm{NaCl}$, and then dried over anhyd. $\mathrm{MgSO}_{4}$. Column 
chromatography $\left(9: 1\right.$, pentane- $\left.\mathrm{Et}_{2} \mathrm{O}\right)$ afforded $1.97 \mathrm{~g}(91 \%)$ of $5 \mathrm{a}$ as a colorless liquid. [5a: ${ }^{1} \mathrm{H}\left(\mathrm{CDCl}_{3}\right) ; \delta 0.82(3 \mathrm{H}, \mathrm{d}, J=8 \mathrm{~Hz}), 0.85-0.98(6 \mathrm{H}, \mathrm{m}), 1.19$ $(2 \mathrm{H}, \mathrm{m}), 1.2-1.58(7 \mathrm{H}, \mathrm{m}), 1.68\left(1 \mathrm{H}, \mathrm{s} . \mathrm{D}_{2} \mathrm{O}\right.$ exchangeable $), 3.41(1 \mathrm{H}$, $\mathrm{m}) ;{ }^{13} \mathrm{C}\left(\mathrm{CDCl}_{3}\right) \delta 74.83,39.94,34.17,28.42,25.98,22.76,14.02,13.11$, $11.65 \mathrm{ppm}$. Anal. calcd. for $\mathrm{C}_{9} \mathrm{H}_{20} \mathrm{O}: \mathrm{C}, 74.92 ; \mathrm{H}, 13.98$; found: $\mathrm{C}, 74.77$; $\mathrm{H}, 13.88$.] The use of $\mathrm{D}-(-)$-DET in the asymmetric epoxidation followed by the same synthetic procedure renders the antipode, $5 \mathrm{~b}(1.97 \mathrm{~g}, 91 \%$ yield, $87 \%$ ee. Anal. calcd. $\mathrm{C}_{2} \mathrm{H}_{20} \mathrm{O}: \mathrm{C}, 74.92 ; \mathrm{H}, 13.98$; found: $\mathrm{C}, 74.89 ; \mathrm{H}, 13.75$ ).

The corresponding anti-alcohols, $\mathbf{5 c}$ and $\mathbf{5 d}$, were synthesized according to the same synthetic scheme except (2E)-2-pentenol 6 was used as starting material (Figure 2). The alkenol 6 was prepared by hydride reduction of 3-pentynol (Aldrich Chemical Co.) in $83 \%$ yield (Brandsma, 1988) [6: ${ }^{1} \mathrm{H}\left(\mathrm{CDCl}_{3}\right)$ : $\delta 1.0(3 \mathrm{H}, \mathrm{t}, J=7 \mathrm{~Hz}), 1.8\left(1 \mathrm{H}\right.$, brs, $\mathrm{D}_{2} \mathrm{O}$ exchangeable $), 2.05(2 \mathrm{H}, \mathrm{m}$, $J=8,1.2 \mathrm{~Hz}), 4.05(2 \mathrm{H}, \mathrm{d}, J=8 \mathrm{~Hz}), 5.60(1 \mathrm{H}, \mathrm{dt}, J=13.8,1.3 \mathrm{~Hz})$, $5.75(1 \mathrm{H}, \mathrm{dt}, J=13.8,1.3 \mathrm{~Hz}) ;{ }^{13} \mathrm{C}\left(\mathrm{CDCl}_{3}\right) \delta 134.83,127.94,63.68,25.14$, $13.30 \mathrm{ppm}]$.

(2S,3S)-2,3-Epoxy-pentan-1-ol (7a). This was prepared according to the procedure used for $2 \mathrm{a}$. Thus, $0.5 \mathrm{~g}$ of $4 \mathrm{~A}$ powdered, activated molecular sieves and $5.7 \mathrm{ml}(5.42 \mathrm{~g}, 19 \mathrm{mmol})$ of titanium(IV) isopropoxide in $150 \mathrm{ml}$ of dry $\mathrm{CH}_{2} \mathrm{Cl}_{2}$ were cooled to $-78^{\circ} \mathrm{C}$ in an acetone-Dry Ice bath. To this was added via syringe $3.9 \mathrm{ml}(4.69 \mathrm{~g}, 23 \mathrm{mmol})$ of diethyl $(2 R, 3 R)$-tartrate [L-(+)-DET]

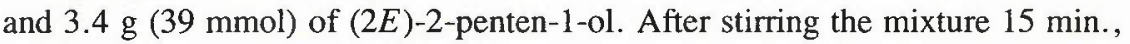
$12 \mathrm{ml}(68 \mathrm{mmol})$ of $5.7 \mathrm{M}$ anhydrous tert-butyl hydroperoxide in $\mathrm{CH}_{2} \mathrm{Cl}_{2}$ (precooled to $-20^{\circ} \mathrm{C}$ ) was added dropwise. After the reaction had warmed up to $-20^{\circ} \mathrm{C}$, it was stirred at this at this temperature for $4 \mathrm{hr}$ and monitored by TLC ( $2: 8$, pentane:ether; $R_{f}=0.39$ ). Nonaqueous work-up followed by column chromatography gave $7 \mathbf{a}(1.70 \mathrm{~g}, 43 \%$ yield, $96 \%$ ee) as a colorless liquid [7a: ${ }^{1} \mathrm{H}\left(\mathrm{CDCl}_{3}\right): \delta 0.96(3 \mathrm{H}, \mathrm{t}, J=8 \mathrm{~Hz}), 1.52(2 \mathrm{H}, \mathrm{m}), 2.80(2 \mathrm{H}, \mathrm{t}, J=$ $8 \mathrm{~Hz}), 3.02\left(1 \mathrm{H}\right.$, brs, $\mathrm{D}_{2} \mathrm{O}$ exchangeable $), 3.45(1 \mathrm{H}, \mathrm{dd}, J=4,8 \mathrm{~Hz}), 3.70$ $(1 \mathrm{H}, \mathrm{dd}, J=8,2 \mathrm{~Hz}) ;{ }^{13} \mathrm{C}\left(\mathrm{CDCl}_{3}\right) \delta 62.05,58.32,57.10,24.41,13.91$ ppm.] 7b (1.64 g, $41 \%$ yield, $96 \%$ ee) was prepared following the same procedure using diethyl $(2 S, 3 S)$-tartrate [D-( -$)$-DET].

(2R,3R)-3-Methyl-pentane-1,2-diol (8a). $1.18 \mathrm{~g}, 59 \%$ yield, $96 \%$ ee. ${ }^{1} \mathrm{H}$
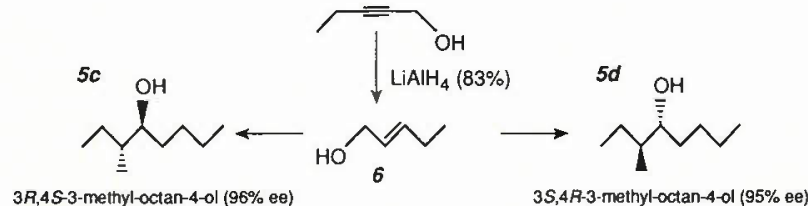

3S,4R-3-methyl-octan-4-ol (95\% ee)

FIG. 2. Scheme for the synthesis of $(3 S, 4 R)$ - and $(3 R, 4 S)$-3-methyl-octan-4-ol. 
$\left(\mathrm{CDCl}_{3}\right): \delta 0.86(3 \mathrm{H}, \mathrm{t}, J=8 \mathrm{~Hz}), 0.91(3 \mathrm{H}, \mathrm{d}, J=8 \mathrm{~Hz}), 1.18(1 \mathrm{H}, \mathrm{m})$, $1.40(2 \mathrm{H}, \mathrm{m}), 3.12\left(2 \mathrm{H}\right.$, brs, $\mathrm{D}_{2} \mathrm{O}$ exchangeable $), 3.40(2 \mathrm{H}, \mathrm{m}), 3.55(1 \mathrm{H}$, $\mathrm{m}$ ); CI-MS $\mathrm{m} / \mathrm{z}$ (relative intensity): $119\left(\mathrm{M}^{+}+1,45\right)$. 8b: $1.28 \mathrm{~g}, 67 \%$ yield, $95 \%$ ee.

(2R,3R)-3-Methyl-1-tosyloxy-pentan-2-ol (9a). $1.52 \mathrm{~g}, 60 \%$ yield, ' $\mathrm{H}$ $\left(\mathrm{CDCl}_{3}\right): \delta 0.84(3 \mathrm{H}, \mathrm{d}, J=8 \mathrm{~Hz}), 0.86(3 \mathrm{H}, \mathrm{t}, J=8 \mathrm{~Hz}), 1.22(1 \mathrm{H}, \mathrm{m})$, $1.45(2 \mathrm{H}, \mathrm{m}), 2.0\left(1 \mathrm{H}, \mathrm{brs}, \mathrm{D}_{2} \mathrm{O}\right.$ exchangeable $), 3.64(1 \mathrm{H}, \mathrm{dt}, J=8,4 \mathrm{~Hz})$, $3.94(1 \mathrm{H}, \mathrm{dd}, J=8,2.5 \mathrm{~Hz}), 4.01(-1 \mathrm{H}, \mathrm{dd}, J=8,1.5 \mathrm{~Hz}) ; 7.30(2 \mathrm{H}, \mathrm{d}$, $J=8 \mathrm{~Hz}) ; 7.75(2 \mathrm{H}, \mathrm{d}, J=8 \mathrm{~Hz}) ; \mathrm{CI}-\mathrm{MS} \mathrm{m} / \mathrm{z}$ (relative intensity): 273 $\left(\mathrm{M}^{+}+1,100\right) .9 \mathrm{~b}: 1.71 \mathrm{~g}, 62 \%$ yield.

(3S, 4R)-3-Methyl-octan-4-ol (5d). $0.68 \mathrm{~g}, 85 \%$ yield, $96 \%$ ee. ${ }^{1} \mathrm{H}\left(\mathrm{CDCl}_{3}\right)$ : $\delta 0.81(3 \mathrm{H}, \mathrm{d}, J=8.1 \mathrm{~Hz}), 0.84-1.0(6 \mathrm{H}, \mathrm{m}), 1.20(2 \mathrm{H}, \mathrm{m}), 1.22-1.60(7 \mathrm{H}$, $\mathrm{m}), 1.70\left(1 \mathrm{H}\right.$, brs, $\mathrm{D}_{2} \mathrm{O}$ exchangeable $), 3.45(1 \mathrm{H}, \mathrm{m}) ;{ }^{13} \mathrm{C}\left(\mathrm{CDCl}_{3}\right) \delta 75.71$, $40.50,33.07,28.25,24.55,22.76,14.70,13.11,11.82 \mathrm{ppm}$. Anal. calcd. for $\mathrm{C}_{9} \mathrm{H}_{20} \mathrm{O}: \mathrm{C}, 74.92 ; \mathrm{H}, 13.98$, found: $\mathrm{C}, 74.76 ; \mathrm{H}, 14.07$. 5d: $0.76 \mathrm{~g}, 84 \%$ yield, $95 \%$ ee. Anal. calcd. for $\mathrm{C}_{9} \mathrm{H}_{20} \mathrm{O}: \mathrm{C}, 74.92 ; \mathrm{H}, 13.98$, found: $\mathrm{C}, 75.06$; H, 14.01, Figure 2.

Synthesis of Cruentol Stereoisomers

$(4 R, 5 R)-, \quad(4 S, 5 S)-, \quad(4 R, 5 S)-$, and $(4 S, 5 R)-5$-methyl-octan-4-ol $(R, R-$, $S, S-, R, S-$, and $S, R$-cruentol) were synthesized according to a method modified from Nakagawa and Mori (1984), followed by Mitsunobu reaction (Mitsunobu, 1981) of the corresponding anti-isomers (Figure 3 ).

(2S,3S)-2,3-Epoxy-hexan-1-ol (11a). This was prepared according to the procedure employed for $2 \mathrm{a}$. Thus, $1 \mathrm{~g}$ of $4 \mathrm{~A}$ powdered, activated molecular sieves and $8.4 \mathrm{ml}(8.79 \mathrm{~g}, 31 \mathrm{mmol})$ of titanium(IV) isopropoxide in $250 \mathrm{ml}$ of dry $\mathrm{CH}_{2} \mathrm{Cl}_{2}$ were cooled to $-78^{\circ} \mathrm{C}$ in an acetone-Dry Ice bath. Then via syringe was added $6.3 \mathrm{ml}(5.23 \mathrm{~g}, 25 \mathrm{mmol})$ of diethyl $(2 R, 3 R)$-tartrate [L-(+)-DET] and $6.1 \mathrm{ml}(5.2 \mathrm{~g}, 52 \mathrm{mmol}$ ) of (2E)-2-hexen-1-ol 10 (Aldrich Chemical Co.). Stirring of the mixture was followed by dropwise addition of $18 \mathrm{ml}(0.11 \mathrm{~mol})$ of $6.2 \mathrm{M}$ anhydrous tert-butyl hydroperoxide in $\mathrm{CH}_{2} \mathrm{Cl}_{2}$ (precooled to $-20^{\circ} \mathrm{C}$ ). The reaction was allowed to warm to $-20^{\circ} \mathrm{C}$ with stirring and was stirred at this temperature for $3 \mathrm{hr}$ while it was monitored by TLC $(4: 6$, hexane-ether; $R_{f}=0.19$ ). Ferrous sulfate/tartaric acid work-up (Gao et al., 1987) followed by column chromatography gave $11 \mathrm{a}(4.82 \mathrm{~g}, 80 \%$ yield, $95 \%$ ee) as a colorless liquid, which crystallized as white needles at $-20^{\circ} \mathrm{C}$. [11a: ${ }^{1} \mathrm{H}\left(\mathrm{CDCl}_{3}\right): \delta 0.96$ $(3 \mathrm{H}, \mathrm{t}, J=7.6 \mathrm{~Hz}), 1.48(2 \mathrm{H}, \mathrm{m}), 1.54(2 \mathrm{H}, \mathrm{m}), 1.80\left(1 \mathrm{H}, \mathrm{brs}, \mathrm{D}_{2} \mathrm{O}\right.$ exchangeable), $2.92(2 \mathrm{H}, \mathrm{m}), 3.60(1 \mathrm{H}, \mathrm{dd}, J=5,10 \mathrm{~Hz}), 3.90(1 \mathrm{H}$, dd, $J=10,2.5 \mathrm{~Hz}) ;{ }^{13} \mathrm{C}\left(\mathrm{CDCl}_{3}\right) \delta 61.76,58.34,55.81,33.57,19.23,13.84$ ppm.] $11 \mathrm{~b}$ (4.94 g, 82\% yield, 95\% ee) was prepared following the same procedure but employing diethyl $(2 S, 3 S)$-tartrate [D-(-)-DET]. 


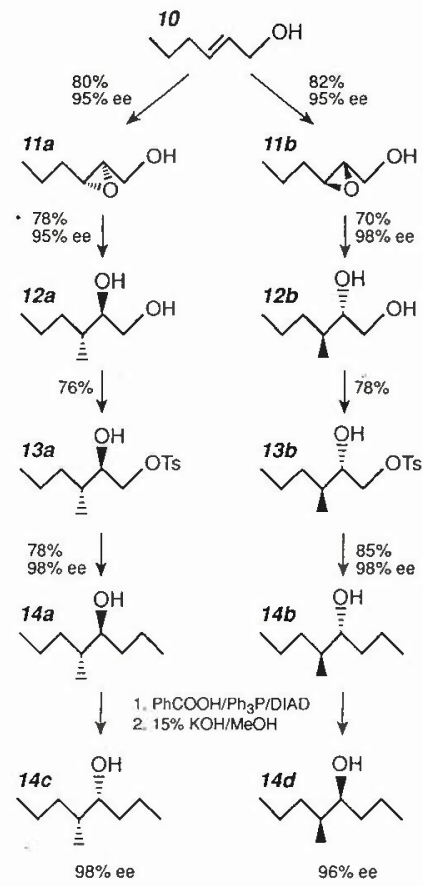

FIG. 3. Scheme for the synthesis of all four stereoisomers of 5-methyl-octan-4-ol.

(2R,3R)-3-Methyl-hexane-1,3-diol (12a). This was prepared according to the procedure employed for 3a. Thus, to $4.80 \mathrm{~g}(0.04 \mathrm{~mol})$ of $11 \mathrm{a}$ in $250 \mathrm{ml}$ of dry pentane cooled to $-50^{\circ} \mathrm{C}$ was added dropwise $11.9 \mathrm{ml}(8.64,0.11 \mathrm{~mol})$ of neat $\mathrm{AlMe}_{3}$. This was followed by $16 \mathrm{ml}$ of $2.49 \mathrm{M} n$-butyllithium $(0.04$ $\mathrm{mol}$ ). After stirring $20 \mathrm{~min}$, the cooling bath was removed and the flask allowed to warm to room temperature. The reaction was monitored by GC and TLC ( $2: 8$, hexane-ethyl acetate, $R_{f}=0.33$ ) and was complete after $30 \mathrm{~min}$. After quenching with $3 \mathrm{M} \mathrm{HCl}$ at $0^{\circ} \mathrm{C}$ and separation of the two phases, the aqueous layer was extracted with ether $(3 \times 40 \mathrm{ml})$, dried over anhyd. $\mathrm{MgSO}_{4}$, and concentrated in vacuo. Purification by column chromatography afforded 12a $(4.26 \mathrm{~g}, 78 \%$ yield, $95 \% \mathrm{ee})$ as a colorless liquid, which crystallized as a white solid at $-20^{\circ} \mathrm{C}$. [12a: ${ }^{1} \mathrm{H}\left(\mathrm{CDCl}_{3}\right): \delta 0.88(3 \mathrm{H}, \mathrm{t}, J=10 \mathrm{~Hz}), 0.90(3 \mathrm{H}, \mathrm{d}$, $J=10 \mathrm{~Hz}), 1.14(1 \mathrm{H}, \mathrm{m}), 1.25(1 \mathrm{H}, \mathrm{m}), 1.46(1 \mathrm{H}, \mathrm{m}), 1.60(1 \mathrm{H}, \mathrm{m})$, 2.10 (1 H, brs, $\mathrm{D}_{2} \mathrm{O}$ exchangeable), 2.24 ( $1 \mathrm{H}$, brs, $\mathrm{D}_{2} \mathrm{O}$ exchangeable), 3.50 $(2 \mathrm{H}, \mathrm{m}), 3.70(1 \mathrm{H}, \mathrm{m}) ;{ }^{13} \mathrm{C}\left(\mathrm{CDCl}_{3}\right) \delta 76.28,64.66,35.94,34.68,20.06$, 15.14, 14.26); CI-MS $m / z$ (relative intensity): $119\left(\mathrm{M}^{+}+1,40.\right] 12 b: 3.91 \mathrm{~g}$, $70 \%$ yield, $98 \%$ ee.

(2R,3R)-3-Methyl-1-tosyloxy-hexan-2-ol (13a). After purification by col- 
umn chromatography (6:4, pentane-ether, $\left.R_{f}=0.45\right), 13 \mathrm{a}(6.54 \mathrm{~g}, 76 \%$ yield) was obtained as a pale yellow oil, ${ }^{1} \mathrm{H}\left(\mathrm{CDCl}_{3}\right): \delta 0.86(6 \mathrm{H}, \mathrm{m}), 1.18(2 \mathrm{H}, \mathrm{m})$, $1.40(2 \mathrm{H}, \mathrm{m}), 1.60(1 \mathrm{H}, \mathrm{m}), 1.90\left(1 \mathrm{H}\right.$, brs $\mathrm{D}_{2} \mathrm{O}$ exchangeable $), 2.48(3 \mathrm{H}, \mathrm{s})$, $3.64(1 \mathrm{H}, \mathrm{m}), 3.98(1 \mathrm{H}, \mathrm{dd}, J=12,8 \mathrm{~Hz}), 4.10(1 \mathrm{H}, \mathrm{dd}, J=12,4 \mathrm{~Hz})$, $7.38(2 \mathrm{H}, \mathrm{d}, J=8 \mathrm{~Hz}), 7.80(2 \mathrm{H}, \mathrm{d}, J=8 \mathrm{~Hz}) ;{ }^{13} \mathrm{C}\left(\mathrm{CDCl}_{3}\right) \delta 144.99,132.5$, $129.92,127.93,73.43,72.66,35.54,34.15,21.26,19.96,15.10,14.16 \mathrm{ppm}$. 13b: $6.09 \mathrm{~g}, 78 \%$ yield.

(4S,5R)-5-Methyl-octan-4-ol (14a). This was prepared by the route used for 5a except that ethyl magnesium bromide (Aldrich Chemical Co.) ( $3 \mathrm{M}$ solution in $\mathrm{Et}_{2} \mathrm{O}$ ) was used. After purification by column chromatography $(9: 1$, pentane-ether, $\left.R_{f}=0.08\right), 14 a(2.74 \mathrm{~g}, 78 \%$ yield, $98 \%$ ee), was obtained as a colorless liquid, which crystallized as a white solid at $-20^{\circ} \mathrm{C},{ }^{\prime} \mathrm{H}\left(\mathrm{CDCl}_{3}\right)$ : $\delta 0.90(3 \mathrm{H}, \mathrm{t}, J=8 \mathrm{~Hz}), 0.92(3 \mathrm{H}, \mathrm{d}, J=8 \mathrm{~Hz}), 0.94(3 \mathrm{H}, \mathrm{t}, J=8 \mathrm{~Hz})$, $1.10(1 \mathrm{H}, \mathrm{m}), 1.24(1 \mathrm{H}, \mathrm{m}), 1.32(1 \mathrm{H}, \mathrm{m}), 1.40(4 \mathrm{H}, \mathrm{m}), 1.50(1 \mathrm{H}, \mathrm{m})$, $1.70\left(1 \mathrm{H}\right.$, brs, $\mathrm{D}_{2} \mathrm{O}$ exchangeable $), 3.48(1 \mathrm{H}, \mathrm{m}) ;{ }^{13} \mathrm{C}\left(\mathrm{CDCl}_{3}\right) \delta 75.82,38.61$, $35.64,34.17,20.42,19.28,15.24,14.34,14.13 \mathrm{ppm}$; CI-MS $\mathrm{m} / \mathrm{z}$ (relative intensity): $127(100)\left(\mathrm{M}^{+}-\mathrm{H}_{2} \mathrm{O}\right)$ : Anal. calcd. for $\mathrm{C}_{9} \mathrm{H}_{20} \mathrm{O}: \mathrm{C}, 74.92 ; \mathrm{H}, 13.98$, found: C, 75.16; H, 14.11. 14b: $2.73 \mathrm{~g}, 85 \%$ yield, $98 \%$ ee; Anal. calcd. for $\mathrm{C}_{9} \mathrm{H}_{20} \mathrm{O}: \mathrm{C}, 74.92 ; \mathrm{H}, 13.98$, found: $\mathrm{C}, 73.87 ; \mathrm{H}, 14.08$.

[(4R,5R)-5-Methyl-4-octyl)]benzoate (15a). Triphenylphosphine $(9.97 \mathrm{~g}$, $38 \mathrm{mmol})$ and $14 a(2.74 \mathrm{~g}, 19 \mathrm{mmol})$ in $30 \mathrm{ml}$ of dry benzene were added via cannula to diisopropyl azodicarboxyate $(7.68 \mathrm{~g}, 7.5 \mathrm{ml}, 38 \mathrm{mmol}$ ) (Aldrich Chemical Co.) and benzoic acid (4.64 g, $38 \mathrm{mmol})$ in $45 \mathrm{ml}$ dry benzene. After stirring overnight at room temperature, pentane was added, at which point a white precipitate formed. The reaction mixture was filtered through a Florisil pad and concentrated under pressure. Purification by column chromatography $\left(9: 1\right.$, pentane-ether, $\left.R_{f}=0.61\right)$ afforded $15 \mathrm{a}(2.35 \mathrm{~g}, 50 \%$ yield $)$ as a pale yellow liquid. Unreacted alcohol was recovered. [15a: ${ }^{1} \mathrm{H}\left(\mathrm{CDCl}_{3}\right): \delta 0.88$ $(3 \mathrm{H}, \mathrm{t}, J=9 \mathrm{~Hz}), 0.98(3 \mathrm{H}, \mathrm{t}, J=9 \mathrm{~Hz}), 1.00(3 \mathrm{H}, \mathrm{d}, J=9 \mathrm{~Hz}), 1.10$ $(1 \mathrm{H}, \mathrm{m}), 1.38,(5 \mathrm{H}, \mathrm{m}), 1.58(1 \mathrm{H}, \mathrm{m}), 1.70(1 \mathrm{H}, \mathrm{m}), 1.80(1 \mathrm{H}, \mathrm{m}), 5.10$ $(1 \mathrm{H}, \mathrm{m}), 7.40(2 \mathrm{H}, \mathrm{dd}, J=9,2 \mathrm{~Hz}), 7.54(1 \mathrm{H}, \mathrm{ddd}, J=9,2 \mathrm{~Hz}), 8.04$ $(2 \mathrm{H}, \mathrm{dd}, J=9,2 \mathrm{~Hz}) ;{ }^{13} \mathrm{C}\left(\mathrm{CDCl}_{3}\right) \delta 166.37,132.62,130.98,129.56,128.29$, $77.74,36.27,35.41,33.77,20.34,19.07,14.48,14.22,14.01$ ppm; CI-MS $\mathrm{m} / \mathrm{z}$ (relative intensity): $127\left(\mathrm{M}^{+}-\mathrm{C}_{6} \mathrm{H}_{5}-\mathrm{CO}, 100\right)$.] 15b: $2.70 \mathrm{~g}, 57.3 \%$ yield.

(4R,5R)-5-Methyl-octan-4-ol (14c). To a $15 \% \mathrm{KOH}$ solution of methanol was added 15a $(1.30 \mathrm{~g}, 52 \mathrm{mmol})$. After stirring the mixture overnight, it was quenched with water and extracted with $\mathrm{Et}_{2} \mathrm{O}(3 \times 30 \mathrm{ml})$. The ether extracts were washed with dilute $\mathrm{HCl}$ and saturated $\mathrm{NaCl}$ and then dried over anhyd. $\mathrm{MgSO}_{4}$. Concentration in vacuo and column chromatography $(9: 1$, pentaneether, $\left.R_{f}=0.13\right)$ gave $14 \mathrm{c}(0.71 \mathrm{~g}, 95 \%$ yield, $98 \% \mathrm{ee})$ as a colorless liquid. [14c: ' $\mathrm{H}\left(\mathrm{CDCl}_{3}\right): \delta 0.89(3 \mathrm{H}, \mathrm{d}, J=8 \mathrm{~Hz}), 0.92(3 \mathrm{H}, \mathrm{t}, J=8 \mathrm{~Hz}), 0.95$ $(3 \mathrm{H}, \mathrm{t}, J=8 \mathrm{~Hz}), 1.12(1 \mathrm{H}, \mathrm{m}), 1.24(1 \mathrm{H}, \mathrm{m}), 1.33(1 \mathrm{H}, \mathrm{m}), 1.39(5 \mathrm{H}$, 
m), $1.48(1 \mathrm{H}, \mathrm{m}), 3.40(1 \mathrm{H}, \mathrm{m}) ;{ }^{13} \mathrm{C}\left(\mathrm{CDCl}_{3}\right) \delta 75.04,38.7036 .73,35.73$, $20.51,19.51,15.33,14.38,13.65 \mathrm{ppm}$; Anal. calcd. for $\mathrm{C}_{9} \mathrm{H}_{20} \mathrm{O}: \mathrm{C}, 74.92$; $\mathrm{H}, 13.98$, found: $\mathrm{C}, 74.74 ; \mathrm{H}, 13.84$.] 14d: $0.75 \mathrm{~g}, 89 \%$ yield, $96 \%$ ee; Anal. calcd. for $\mathrm{C}_{9} \mathrm{H}_{20} \mathrm{O}: \mathrm{C}, 74.92 ; \mathrm{H}, 13.98$; found: $\mathrm{C}, 74.69 ; \mathrm{H}, 13.81$.

The enantiomeric excesses of $\mathbf{5 a}(93 \%), \mathbf{5 b}(87 \%), \mathbf{5 c}(96 \%), \mathbf{5 d}(95 \%)$, 14a $(98 \%), 14 \mathrm{~b}(98 \%), \mathbf{1 4 c}(98 \%)$, and $14 \mathrm{~d}(96 \%)$ and their corresponding intermediates except epoxides were determined by GC analyses on the Cyclodex-B column and by formation of the $O$-acetyllactyl methyl esters (Slessor et al., 1985). Enantiomeric excesses of epoxides $2 \mathbf{a}(85 \%), \mathbf{2 b}(88 \%), \mathbf{7 a}$ $(96 \%), 7 \mathbf{b}(96 \%), \mathbf{1 1 a}(95 \%)$, and $\mathbf{1 1 b}(95 \%)$ were determined by GC analysis of corresponding $O$-acetyllactyl methyl esters (Slessor et al., 1985) on a DB-23 column. Racemic 3-methyl-octan-4-ol and 5-methyl-octan-4-ol were synthesized as previously described (Gries et al., 1993; Weissling et al., 1994).

\section{Field Experiments}

African Palm Weevil. A six-replicate, five-treatment field experiment in a 10-year-old oil palm stand (La Me Research Station, Côte d'Ivoire) tested attraction of palm tissue $(250 \mathrm{~g})$ alone or in combination with either stereoisomeric, $(S, S)-,(R, R)$-, or $(S, S)$ - plus $(R, R)$-phoenicol. Traps (Oehlschlager et al., 1993) were attached at breast height to oil palms in randomized blocks with traps at 27 -m intervals and blocks $81 \mathrm{~m}$ apart. $(S, S)$ - and $(R, R)$-phoenicol were released at $0.5 \mathrm{mg} /$ day (at $25^{\circ} \mathrm{C}$ ) from a $1.5-\mathrm{ml}$ polyethylene centrifuge tube with two 2 -mm holes below the top. Racemic phoenicol was dispensed at $2 \mathrm{mg} /$ day (at $25^{\circ} \mathrm{C}$ ) from four 1.5 -ml polyethylene centrifuge tubes. Fresh palm tissue in each trap was treated with insecticidal (biodegradable) Evisect " $S$ " $(0.3 \%$ thiocyclamhydrogenoxalate in water) to retain captured weevils (Gries et al., 1993, 1994).

Trap catch data were subjected to analysis of variance followed by Scheffé test for comparisons of means (Zar, 1984).

Palmetto Weevil. A 12-replicate, four-treatment experiment in the same location as for weevil collection tested attraction of Sabal palmetto tissue (1.5 $\mathrm{kg}$ ) alone or in combination with either stereoisomeric, $(S, S)$ - or $(R, R)$-cruentol. Traps (Weissling et al., 1994) were secured on the ground in randomized complete blocks with traps at 20-m intervals and blocks at least $50 \mathrm{~m}$ apart. Unlike Weissling's trap, a tapered, inverted white plastic container (4.9 liter) with a screened lid was suspended in the mouth of the bucket by a capped PVC pipe $(1.3 \mathrm{~cm}$ diameter) from which pheromone release devices were hung. $(S, S)$ - or $(R, R)$-cruentol were released at $0.06 \mathrm{mg} / \mathrm{day}\left(\right.$ at $\left.25^{\circ} \mathrm{C}\right)$ from one and stereoisomeric cruentol from four bottom-sealed $1-\mu \mathrm{l}$ microcapillary tubes (Drummond Scientific Co., Broomall, Pennsylvania) placed in bottom-sealed microhematocrit tubes (length 75 mm, ID 1.1-1.2 mm; Fisher Scientific, Pittsburgh, Penn- 
sylvania). Hematocrit tubes were placed into polypropylene centrifuge tubes (Corning Glass Works, Corning, New York, with 6-mm holes drilled $1.8 \mathrm{~cm}$ from the top). Trap catch data were subjected to square root $(x+0.5)$ transformation and ANOVA (SAS Institute, 1990) followed by Waller-Duncan $k$-ratio $t$ test to test differences between means $(P \leq 0.05)$.

0

$+$

\section{RESULTS AND DISCUSSION}

Many coleopteran pheromones are optically active (Seybold, 1993; Leal and Mochizuki, 1993; Bestmann and Vostrowsky, 1988; Evershed, 1988; Borden, 1985, and literature cited therein). Enantioselective production of and response to pheromones contribute to species specificity of semiochemical communication (Borden et al., 1976, 1980; Brand et al., 1979; Birch et al., 1980; Payne et al., 1982; Oehlschlager et al., 1987; Pierce et al., 1987; Birch, 1984; Byers, 1989). The presence of nonnatural (non-beetle-produced) enantiomers in synthetic pheromones has been demonstrated to interfere with optimal attraction. For instance, the male-produced aggregation pheromone in the southern pine beetle, Dendroctonus frontalis Zimm., $(1 R, 5 S, 7 S)-(+)$-endo-brevicomin, endo7-ethyl-5-methyl-6,8-dioxa-[3.2.1]octane, markedly enhances the response by both sexes to female-produced frontalin (1,5-dimethyl-6,8-dioxa-[3.2.1]octane), whereas the presence of the antipode in racemic endo-brevicomin interferes with optimal attraction (Vité et al., 1985). In the Japanese beetle, Popillia japonica (N.), female-produced Japonilure, $(R, Z)-(-)-5$-(1-decyl)oxacyclopentan-2-one, strongly attracts males, whereas the antipode inhibits responses (Tumlinson et al., 1977). In the scarab beetle, Anomala cuprea only the $(R, Z)-5-(-)$-(oct-1enyl)oxacyclopentan-2-one attracts conspecifics, while the presence of the nonnatural enantiomer reduced attraction (Leal and Mochizuki, 1993). Determination of insect-produced pheromone enantiomer(s) and/or stereoisomers is required to fully elucidate the chemical communication system for a target insect and to implement efficient pheromone-based monitoring and/or management. In this study, we confirmed the chirality of weevil-produced phoenicol (Gries et al., 1993; Perez et al., 1993; Mori et al., 1993), determined chirality of weevilproduced cruentol (Weissling et al., 1994), and field tested weevil attraction to natural and nonnatural stereoisomers.

Of several methods available to prepare the target chiral $\alpha$-methyl secondary alcohols, the Sharpless asymmetric epoxidation combined with diastereoselective ring opening was the most appealing (Gao et al., 1987; Hill et al., 1985; for use of trimethylaluminum and organocuprates: Pfaltz and Mattenberger, 1982; Suzuki et al., 1982; Takano et al., 1989; Vaccaro et al., 1992; Miyashita et al., 1993). This strategy allowed the use of inexpensive reagents and the synthesis of all four stereoisomers from the same starting material. 
Sharpless asymmetric epoxidation has been previously used for the synthesis of the stereoisomers of the elm bark beetle pheromone, 4-methyl-heptan3-ol (Nakawaga and Mori, 1984). In contrast to this previous synthesis, preparation of phoenicol and cruentol used 0.5 equivalents of catalyst in the presence of a $4 \mathrm{~A}$ molecular sieve coupled with addition of the oxidizing agent at $-78^{\circ} \mathrm{C}$ to increase the optical purity of the initial epoxide product. Epoxidations were maintained at $-20^{\circ} \mathrm{C}$ until $97-98 \%$ conversion was obtained for $\mathbf{2 a}$ and $\mathbf{2 b}$ (two days) as well as for 7a, 7b, 11a, and 11b (3-4 hr). Although reactions are not reported for diisopropropyl tartrate, higher enantioselectivities were achieved with diethyl tartrate. Nonaqueous work-up (Gao et al., 1987) followed by flash chromatography was used in the synthesis of the C-5 epoxides. Separation of the tartrate from the C-5 epoxides required two or more chromatographic cycles, whereas ferrous sulfate/tartaric acid work-up (Gao et al., 1987) followed by a single chromatography cleanly gave the $\mathrm{C}-6$ epoxides. Diasteroselective epoxide ring-opening was conducted with neat $\mathrm{AlMe}_{3}$ instead of a hexane solution of this reagent, as was employed by Nakawaga and Mori. This facilitated completion of the reaction in less than $1 \mathrm{hr}$ compared to two to three days. Work-up via addition of saturated $\mathrm{NaF}$ at $-40^{\circ} \mathrm{C}$ (Suzuki et al., 1982) for 3a, 3b, 8a, and $8 \mathbf{b}$, and $3 \mathrm{M} \mathrm{HCl}$ for $12 \mathrm{a}$ and $\mathbf{1 2 b}$ afforded the corresponding diols after flash chromatography. Quenching with $\mathrm{NaF}$ rather than $\mathrm{HCl}$ improved isolated yields of 3-methyl-1,2-pentanediols, probably due to the high solubility of the diols in water. Products arising from breakage of the $\alpha$-bond or retention of configuration during the cleavage of the $\beta$-epoxide bond were not detected by $\mathrm{GC}$ or ${ }^{1} \mathrm{H}$ NMR analysis. The syn-isomers of phoenicol and cruentol were obtained with moderate optical purities from asymmetric epoxidation of the requisite Z-alkenols. In contrast, Mori and Brevet (1991) and Mori and Harashima (1993) generated chirally pure epoxides through crystallization of derivatives, a process that leads to yields in the range of $40 \%$. The $p$-nitrobenzoates or 3,5-dinitrobenzoates of $\mathbf{2} \mathbf{a}$ and $\mathbf{2 b}$ were oils at room temperature and below.

The syn isomers of 5-methyl-octan-4-ol were obtained in high enantiomeric excess through Mitsunobu (Mitsunobu, 1981; Hughes, 1992, and references cited therein) mediated inversion of configuration of the anti-isomers 14a and 14b. Use of $p$-nitrobenzoic acid- $\mathrm{Ph}_{3} \mathrm{P}$-diethyl azocarboxylate (DEAD) in THF yielded less than $25 \%$ of the corresponding $p$-nitrobenzoates. Successful Mitsunobu conditions ( $\sim 51 \%$ yields) employed benzoic acid- $\mathrm{Ph}_{3} \mathrm{P}$-diisopropyl azocarboxylate (DIAD) and benzene as a solvent (Paquette and Sugimura, 1986; Dai et al., 1988; Dyer and Kishi, 1988). No epimerization or retention of configuration products were observed.

Both stereoisomeric phoenicol and cruentol elute from a polar SP-1000coated, fused silica column in two resolved components. The shorter eluting component coincided with the male-produced pheromone of each weevil and was hypothesized to be the syn diastereoisomer, consisting of coeluting $S, S$ and 
$R, R$ isomers. This assignment was made by analogy with the aggregation pheromone of the smaller European elm bark beetle, Scolytus multistriatus (Marsham), 4-methyl-heptan-3-ol, that also has two stereogenic centers and exists as two diastereoisomeric forms that are separable by GC on a polar Carbowax 20 M column (Pearce et al., 1975). Analysis of stereoselectively prepared syn and anti stereoisomers of phoenicol and cruentol confirmed the assignments. Synthetic $(R, R)$-, $(S, S)$-, $(R, S)$-, and $(S, R)$-phoenicol and cruentol were separated with baseline resolution on a fused silica, Cyclodex-B column. These analyses revealed that male $R$. phoenicis and male $R$. cruentatus produce the $S, S$ stereoisomer of phoenicol (Figure 4) and cruentol (Figure 5), respectively. S. multistriatus also produces the $S, S$ stereoisomer of 4-methyl-heptan-3-ol (Pearce et al., 1975), whereas the large European elm bark beetle, Scolytus scolytus (F.), produces both $(3 S, 4 S)$ - and $(3 R, 4 S)$-4-methyl-heptan-3-ol (Blight et al., 1977, 1978, 1979; Wadhams et al., 1982).

Coupled GC-EAD of synthetic phoenicol (Figure 6) and cruentol (Figure 7) revealed strong antennal responses to weevil-produced $(S, S)$-phoenicol and $(S, S)$-cruentol. Lack of or reduced response to later-eluting stereoisomers can hardly be explained by an antennal refractory period. In GC-EAD recordings with the same Cyclodex-B column, antennae of two Asian palm weevils, $R$. ferrugineus (Oliv.) and $R$. vulneratus (Panz.), distinctively responded to both, closely eluting, $(4 S)$ - and (4R)-4-methyl-nonan-5-one (Perez et al., unpublished). Similarly, in GC-EAD analyses of oil palm volatiles, antennae of male

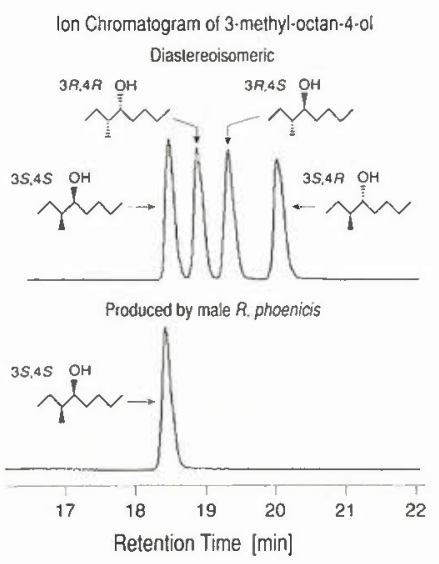

Fig. 4. Selected ion $m / z 127$ chromatogram (Hewlett Packard 5985B) of stereoisomeric and weevil-produced 3-methyl-octan-4-ol. $m / z 127$ was the parent ion $\left[\left(\mathrm{M}^{+}-\mathrm{H}\right) 143\right.$, $\left(\mathrm{M}^{+}-\mathrm{H}-\mathrm{OH}\right)$ 127] of the full-scan mass spectrum in CI mode. (Cyclodex-B column; $90^{\circ} \mathrm{C}$ isothermal; linear flow velocity of carrier gas: $35 \mathrm{~cm} / \mathrm{sec}$; injector temperature: $220^{\circ} \mathrm{C}$ ). 

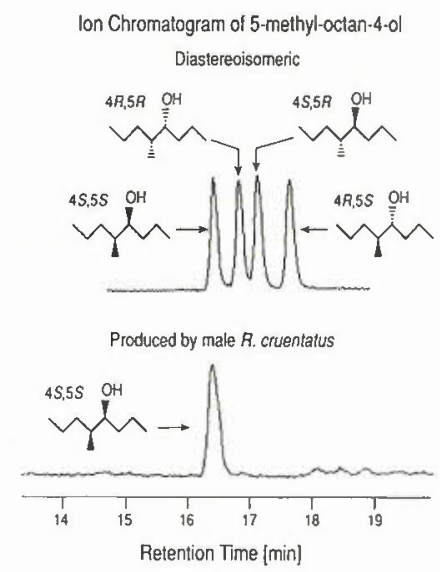

Frg. 5. Selected ion $\mathrm{m} / \mathrm{z} 127$ chromatogram of stereoisomeric and weevil-produced 5-methyl-octan-4-ol. $m / z 127$ was the parent ion [ $\left.\left(\mathrm{M}^{+}-\mathrm{H}\right) 143,\left(\mathrm{M}^{+}-\mathrm{H}-\mathrm{OH}\right) 127\right]$ of the full-scan mass spectrum in $\mathrm{CI}$ mode (instrument and chromatographic conditions as in Figure 4).

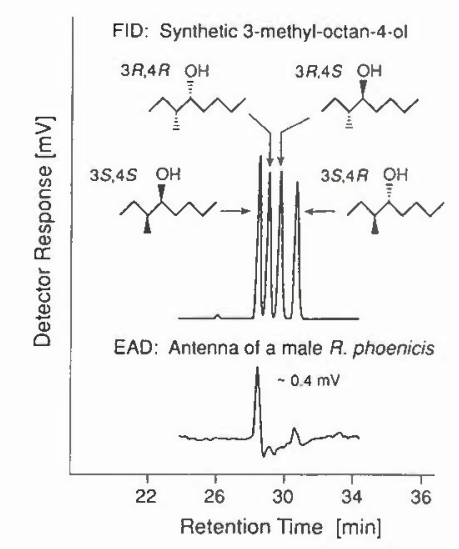

FIG. 6. Representative GC-EAD recording of a female Rhynchophorus phoenicis antenna responding to stereoisomers of 3-methyl-octan-4-ol (Hewlett Packard 5890A; split injection; column and chromatographic conditions as in Figure 4).

and female $R$. phoenicis responded within $2.5 \mathrm{~min}$ to four esters, two of which were barely baseline separated (Gries et al., 1994). Strong antennal activity of the $S, S$, and weak activity of $S, R$ and $R, S$ isomers of the pheromones (Figures 6 and 7) suggest that sensory recognition of the natural $S, S$ stereoisomer is more dependent on the stereochemistry of the methyl than the hydroxy group. 
In field experiments $(S, S)$-phoenicol and $(S, S)$-cruentol strongly synergized attraction of weevils to palm tissue (Figures 8 and 9). Because racemic, stereoisomeric mixtures were as synergistic as $S, S$ isomers, the weakly EAD-active $S, R$ isomers (Figures 6 and 7) neither enhanced nor reduced behavioral activity in the stereoisomeric mixtures (Figures 8 and 9). Lack of strong antennal (Figures 6 and 7) and any behavioral activity (Figures 8 and 9) of nonnatural isomers suggests that sympatric beetles are unlikely to utilize one or more stereoisomers

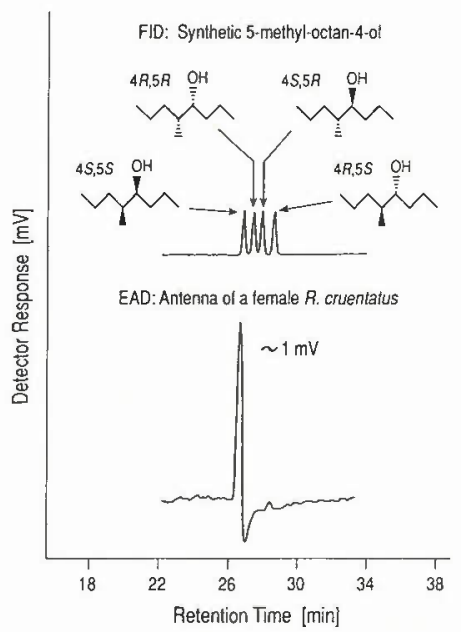

FIG. 7. Representative GC-EAD recording of a female Rhynchophorus cruentatus antenna responding to stereoisomers of 5-methyl-octan-4-ol (Hewlett Packard 5890A; split injection; column and chromatographic conditions as in Figure 4).

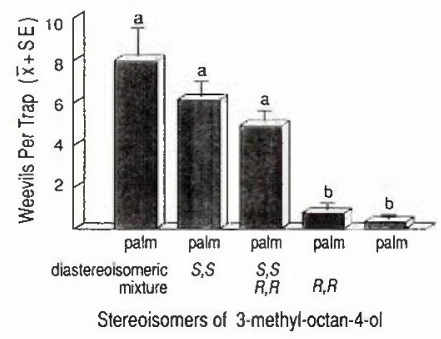

Fig. 8. Mean counts ( + standard error) of male and female $R$. phoenicis in traps baited with $250 \mathrm{~g}$ of chopped oil palm tissue alone and in combination with either stereoisomeric, $(3 S, 4 S)$-, $(3 R, 4 R)$ - or $(3 S, 4 S)$ - plus $(3 R, 4 R)$-phoenicol. La Me Research Station, Côte d'Ivoire; May 6-10, 1993; six blocks. Bars superscripted by the same letter are not significantly different. ANOVA followed by Scheffé test, $P<0.05$. 


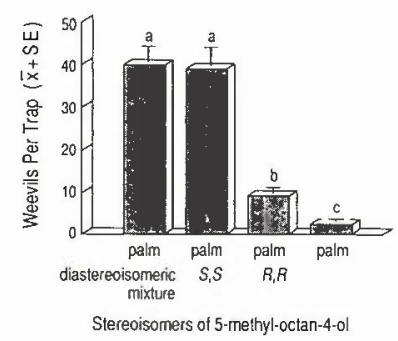

FIG. 9. Mean counts ( + standard error) of male and female $R$. cruentatus in traps baited with $1.5 \mathrm{~kg}$ of chopped Sabal palmetto palm tissue alone and in combination with either stereoisomeric, $(4 S, 5 S)$ - or $(4 R, 5 R)$-cruentol. La Belle, Florida, USA, June 9-16, 1993 ; 12 blocks. Bars superscripted by the same letter are not significantly different. ANOVA followed by Waller-Duncan $k$-ratio $t$ test on square root transformed data $(x+0.5)$, $P \leq 0.05$.

of phoenicol or cruentol as a pheromone. In practice, mixtures of all four stereoisomers of each pheromone could be used in combination with host materials to monitor and/or mass trap $R$. phoenicis and $R$. cruentatus populations.

Acknowledgments-We thank G. Owens for mass spectrometry; the University of Costa Rica for a fellowship to A.L.P.; Thomas J. Weissling, Frank G. Bilz, John Cangiamila, Barbara J. Center, and Mickey Stanaland for field assistance; A. Duda and Sons for providing Sabal palmetto and $R$. cruentatus research site; and Mesmer Zebeyou for hosting G.G. and R.G. in the La Me Research Station, Côte d'Ivoire. A.L.P. thanks Guy V. Lamoureux for helpful discussions. The research was supported by an NSERC operating grant to A.C.O. and by a USDA special grant in Tropical and Subtropical Agriculture CRSR-90-34135-5233 to R.M. G-D.

\section{REFERENCES}

ARN, H., STÄDLER, E., and RAUSCHER, S. 1975. The electroantennographic detector-a selective and sensitive tool in the gas chromatographic analysis of insect pheromones. Z. Naturforsch. 30c:722-725.

Bestmann, H.J., and Vostrowsky, O. 1988. Pheromones of the Coleoptera, pp. 93-183, in E.D. Morgan and N.B. Mandava (eds.). CRC Handbook of Natural Pesticides, Vol. IV, Pheromones Part A. CRC Press, Boca Raton, Florida

BirCH, M.C. 1984. Aggregation in bark beetles, pp. 331-353, in W.J. Bell and R.T. Cardé (eds.) Chemical Ecology of Insects. Chapman and Hall, London.

Birch, M.C., Light, D.L., Wood, D.L., Browne, L.E., Silverstein, R.M., Bergot, B.J., Ohloff, G., West, J.R., and Young, J.C. 1980. Pheromonal attraction and allomonal interruption of Ips pini in California by two enantiomers of ipsdienol. J. Chem. Ecol. 6:703-717.

Blight, M.M., Mellon, F.A., Wadhams, L.J., and Wenham, M.J. 1977. Volatiles associated with Scolytus scolytus beetles on English elm. Experientia 33:845-846.

Blight, M.M., Wadhams, L.J., and Wenham, M.J. 1978. Volatiles associated with unmated Scolytus scolytus beetles on English elm: Differential production of $\alpha$-multistriatin and 4-methyl-3-heptanol, and their activities in a laboratory bioassay. Insect Biochem. 8:135-142. 
Blight, M.M., Wadhams, L.J., and Wenham, M.J. 1979. The stereoisomeric composition of the 4-methyl-3-heptanol produced by Scolytus scolytus and the preparation and biological activity of the four synthetic stereoisomers. Insect Biochem. 9:525-533.

BoRDEN, J.H. 1985. Aggregation pheromones, pp. 257-285, in G.A. Kerkut and L.I. Gilbert (eds.). Comprehensive Insect Physiology, Biochemistry and Pharmacology, Vol. 9. Pergamon Press, Oxford.

Borden, J.H., Chong, L., Mclean, J.A., Slessor, K.N., and Mori, K. 1976. Gnathotrichus sulcatus: Synergistic response to enantiomers of the aggregation pheromone sulcatol. Science 192:894-896.

Borden, J.H., Handley, J.R., Mclean, J.A., Silverstein, R.M., Chong, L., Slessor, K.N., JOHNSTON, B.D., and SCHULER, H.R. 1980. Enantiomer-based specificity in pheromone communication by two sympatric Gnathotrichus species (Coleoptera: Scolytidae). J. Chem. Ecol. 6:445-455.

Brand, J.M., Young, J.C., and Silverstein, R.M. 1979. Insect pheromones: A critical review of recent advances in their chemistry, biology, and application. Fortschr. Chem. Org. Naturst. $37: 1-190$

Brandsma, L. 1988. Preparative Acetylenic Chemistry. Elsevier, Amsterdam. pp. 283-284

Byers, J.A. 1989. Chemical ecology of bark beetles. Experientia 45:271-283.

DAI, L.-X., BO, I. and ZHANG, Y.-Z. 1988. A simple, divergent, asymmetric synthesis of all members of the 2,3,6-trideoxy-3-aminohexose family. J. Am. Chem. Soc. 110:5195-5196.

DYER, U.C., and KISHI, Y. 1988. Synthesis of C-sucrose. J. Org. Chem. 53:3383-3384.

EVERSHED, R.P. 1988. Insect olfaction and molecular structure, pp. 1-33, in E.D. Morgan and N.B. Mandava (eds.). CRC Handbook of Natural Pesticides, Vol. IV, Pheromones Part A CRC Press, Boca Raton, Florida.

Gao, Y., Hanson, R.M., Klunder, J.M., Ko, S.Y., Masamune, H., and Sharpless, K.B. 1987. Catalytic asymmetric epoxidation and kinetic resolution: Modified procedures including in situ derivatization. J. Am. Chem. Soc. 109:5765-5780.

Gries, G., Gries, R., Perez, A.L., Gonzales, L.M., Pierce, H.D., Jr., Oehlschlager, A.C., Rhainds, M., Zebeyou, M., and KouAmE, B. 1994. Ethyl propionate: Synergistic kairomone for African palm weevil, Rhynchophorus phoenicis L. (Coleoptera: Curculionidae). J. Chem. Ecol. 20:889-897.

Gries, G., Gries, R., Perez, A.L., Oehlschlager, A.C., Gonzalez, L.M., Pierce, H.D., Jr., Kouda-Bonafos, M., Zebeyou, M., and NanOU, N. 1993. Aggregation pheromone of the African palm weevil, Rhynchophorus phoenicis (F). Naturwissenschaften 80:90-91.

Hallett, R.H., Gries, G., Gries, R., Borden, J.H., Czyzewska, E., Oehlschlager, A.C., Pierce, H.D., JR., ANGerelli, N.P.D., and Rauf, A. 1993. Aggregation pheromones of two Asian palm weevils, Rhynchophorus ferrugineus and $R$. vulneratus. Naturwissenschaften 80:328-331.

Hill, G., Sharpless, K.B., Exon, C., and Regenye, R. 1985. Enantioselective epoxidation of allylic alcohols: $(2 S, 3 S)$-3-Propyloxiranemethanol (oxiranemethanol, 3-propyl-, $(2 S, 3 S)$-). Org. Synth. 63:66-78.

Hughes, D.L. 1992. The Mitsunobu reaction. Org. React. 42:335-656.

LEAL, W.S., and Mochizuki, F. 1993. Sex pheromone reception in the scarab beetle Anomala cuprea. Enantiomeric discrimination by sensilla placodea. Naturwissenschafien 80:278-281

Mirsunobu, O. 1981. The use of diethyl azocarboxylate and triphenylphosphine in synthesis and transformation of natural products. Synthesis 1981:1-28.

Mryashita, M., Toshimitsu, Y., Siratani, T., and IRIE, H. 1993. Enantioselective synthesis of (-)-serricornin, a sex pheromone of a female cigarette beetle (Lasioderma serricone $\mathrm{F}$.). Tetrahedron Asymm. 4:1573-1578.

Mori, K., and Brevet, J.-L. 1991. Pheromone synthesis: CXXXIII. Synthesis of both the enan- 
tiomers of (3Z,9Z)-cis-6,7-epoxy-3,9-nonadecadiene, a pheromone component of Eranis defoliaria. Synthesis 1991:1125-1129.

Mori, K., and Harashima, S. 1993. Synthesis of $(2 E, 4 E, 6 R, 10 R)-4,6,10,12$-tetramethyl-2,4-tridecadien-7-one (matsuone)-the primary component of the sex pheromone of the three Matsucoccus pine bast scales-and its antipode. Liebigs Ann. Chem. 1993:993-1001.

Mori, K., KiYota, H., and Rochat, D. 1993. Synthesis of the stereoisomers of 3-methyl-4-octanol to determine the absolute configuration of the naturally occurring $(3 S, 4 S)$-isomer isolated as the male-produced aggregation pheromone of the African palm weevil, Rynchophorus phoenicis. Liebigs Ann. Chem. 1993:865-870.

NAKAGAWA, N., and MORI, K. 1984. Synthesis of $(3 S, 4 S)$-4-methyl-3-heptanol and its $(3 S, 4 R)$ isomer employing asymmetric epoxidation coupled with regioselective cleavage of epoxides with trimethylaluminum. Agric. Biol. Chem. 48:2505-2510.

Oehlschlager, A.C., King, G.G.S., Pierce, H.D., Jr., Pierce, A.M., Slessor, K.N., Millar, J.G., and Borden, J.H. 1987. Chirality of macrolides pheromones of grain beetles in the genera Oryzaephilus and Cryptolestes and its implications for species specificity. J. Chem. Ecol. 13:1543-1554.

Oehlschlager, A.C., Pierce, H.D., Jr., Morgan, B., Wimalaratne, P.D.C., Slessor, K.N., King, G.G.S., Gries, G., Gries, R., Borden, J.H., Jiron, L.J., Chinchilla, C.M., and MEXzoN, R.G. 1992. Chirality and field activity of rhynchophorol, the aggregation pheromone of the American palm weevil. Naturwissenschaften 79:134-135.

Oehlschlager, A.C., Chinchilla, C.M., Gonzalez, L.M., Jiron, L.F., Mexzon, R., and MorGAN, B. 1993. Development of a pheromone-based trapping system for Rhynchophorus palmarum (Coleoptera: Curculionidae). J. Econ. Entomol. 86:1381-1392.

Oehlschlager, A.C., Prior, R.N.B., Perez, A.L., Pierce, H.D., Jr., Gries, R., and Gries, G. 1994. Structure, chirality and field testing of male-produced aggregation pheromone for the Asian palm weevil, Rhynchophorus bilineatus (Montr.), (Coleoptera, Curculionidae). J. Chem. Ecol. Submitted.

Paquette, L.A., and Sugimura, T. 1986. Enantiospecific total synthesis and absolute configurational assignment of (-)-puntactin A (antibiotic M95464). J. Am. Chem. Soc. 108:3841-3842.

Payne, T.L., Richerson, J.V., Dickens, J.C., West, J.R., Mori, K., Berisford, C.W., Hedden, R.L., VITÉ, J.P., and BLUM, M.S. 1982. Southern pine beetle: Olfactory receptor and behavior discrimination of enantiomers of the attractant pheromone frontalin. J. Chem. Ecol. 8:873881.

Pearce, G.T., Gore, W.E., Silverstein, R.M., Peacock, J.W., Cuthbert, R.A., Lanier, G.N., and SimEone, J.B. 1975. Chemical attractants for the smaller European elm bark beetle Scolytus multistriatus (Coleoptera: Scolytidae). J. Chem. Ecol. 1:115-124.

Perez, A.L., Gries, R., Gries, G., Hallett, R.H., Oehlschlager, A.C., Pierce, H.D., Jr., Gonzales, L.M., Borden, J.H., and Giblin-Davis, R.M. 1993. Pheromones of Rhynchophorus palm weevils. 10th Annual ISCE Meeting, Tampa, Florida. July 31-August 4.

Pfaltz, A., and Mattenberger, A. 1982. Regioselective opening of $\beta$-alkoxyepoxides with trimethylaluminum. Angew. Chem. Int. Ed. Engl. 21:71-72.

Pierce, A.M., Pierce, H.D., JR, Oehlschlager, A.C., and Borden, J.H. 1987. Response of Oryzaephilus surinamensis and $O$. mercator to the chiral isomers of their macrolide aggregation pheromones. J. Chem. Ecol. 13:1525-1542.

Rochat, D., Malosse, C., Lettere, M., Ducrot, P.-H., Zagatti, P., Renou, M., and Descoins, C. 1991. Male-produced aggregation pheromone of the American palm weevil, Rhynchophorus palmarum L. (Coleoptera: Curculionidae): Collection, identification, electrophysiological activity and laboratory bioassay. J. Chem. Ecol. 17:2127-2141.

Rochat, D., Descoins, C., Malosse, C., Nagnan, P., Zagatti, P., Akamou, F., and Mariau, 
D. 1993. Ecologie chimique des charançons des palmiers, Rhynchophorus spp. (Coleoptera). Oleagineux 48:225-236.

SAS Instirute. 1990. SAS System for Personal Computers, Release 6.04. SAS Institute Inc., Cary, North Carolina.

SEYBOLD, S.J. 1993. The role of chirality in the olfactory-directed aggregation behavior of pine engraver beetles in the genus Ips (Coleoptera: Solytidae). PhD thesis. University of California, Berkeley.

Slessor, K.N., King, G.G.S., Miller, D.R., Winston, M.L., and Cuforth, T.L. 1985. Determination of chirality of alcohol or latent alcohol semiochemicals in individual insects. J. Chem. Ecol. 11:1659-1667.

Still, W.C., KahN, M., and Mitra, A. 1978. Rapid chromatographic technique for preparative separations with moderate resolution. J. Org. Chem. 43:2923-2925.

Suzuki, T., Saimoto, H., Tomioka, H., Oshima, K., and NozaKı, H. 1982. Regio- and stereoselective ring opening of epoxy alcohols with organoaluminum compounds leading to 1,2diols. Tetrahedron Lett. 23:3597-3600.

Takano, S., Yanase, M., and OgaSawaCra, K. 1989. Nucleophilic cleavage of (2S,3S)-3-phenylglycidol. Heterocycles 29:249-252.

Tumlinson, J.H., Klein, M.G., Doolittle, R.E., Ladd, T.L., and Proveaux, A.T. 1977. Identification of the female Japanese beetle sex pheromone: Inhibition of male response by an enantiomer. Science 197:789-792.

Vaccaro, H.A., Levy, D.E., Sawabe, A., Jaetsch, T., and Masamune, S. 1992. Towards the synthesis of calyculin: A synthetic intermediate corresponding to the $\mathrm{C}(26)-\mathrm{C}(37)$ fragment. Tetrahedron Lett. 33:1937-1940.

VITÉ, J.P., BILLING, R.F., WARE, C.W., and MORI, K. 1985. Southern pine beetle: Enhancement or inhibition of aggregation response mediated by enantiomers of endo-brevicomin. Naturwissenschafien 72:99-100.

Wadhams, L.J., ANGST, M.E., and BugGht, M.M. 1982. Responses of the olfactory receptors of Scolytus scolytus (F.) (Coleoptera: Scolytidae) to the stereoisomers of 4-methyl-3-heptanol. J. Chem. Ecol. 8:477-492.

Weissling, T.J., Giblin-Davis, R.M., Gries, G., Gries, R., Perez, A.L., Pierce, H.D., Jr., and Oenlschlager, A.C. 1994. Aggregation pheromone of the Palmetto weevil, Rynchophorus cruentatus (F.) (Coleoptera: Curculionidae). J. Chem. Ecol. 20:505-515.

Zar, J.H. 1984. Biostatistical Analysis. Prentice-Hall, Englewood Cliffs, New Jersey. 718 pp. 